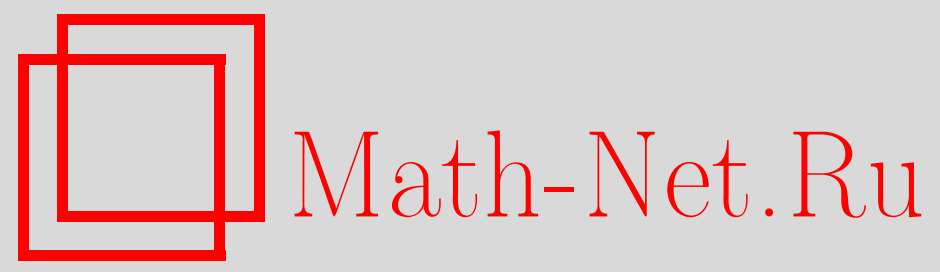

М. Е. Широков, О каналах с конечной $\chi$-пропускной способностью, Теория вероятн. и ее примен., 2008, том 53, выпуск 4, 732-750

DOI: https://doi.org/10.4213/tvp2462

Использование Общероссийского математического портала MathNet.Ru подразумевает, что вы прочитали и согласны с пользовательским соглашением

http://www . mathnet.ru/rus/agreement

Параметры загрузки:

IP : 54.197 .217 .227

26 апреля 2023 г., 06:19:46

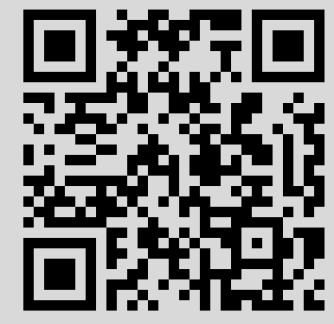


(C) 2008 r.

ШИРОКОВ М. Е.*

\title{
О КАНАЛАХ С КОНЕЧНОЙ $\chi$-ПРОПУСКНОЙ СПОСОБНОСТЬЮ ${ }^{1)}$
}

\begin{abstract}
В статье рассматриваются основные свойства бесконечномерных квантовых каналов, обладающих конечной $\chi$-пропускной способностью. Получены достаточные условия существования оптимальной меры для таких каналов. Рассмотрены примеры каналов, не имеющих оптимальной меры.
\end{abstract}

Ключевые слова и фразы: квантовое состояние, квантовый канал, квантовая энтропия, $\chi$-пропускная способность.

1. Введение. Квантовая теория информации - это быстро развивающаяся в последние годы научная дисциплина, исследующая информационные аспекты квантовомеханических систем, математическим аппаратом которой является теория операторов в гильбертовом пространстве [3].

Одним из основных понятий квантовой теории информации является понятие квантового канала как преобразования состояний квантовой системы. С математической точки зрения квантовые состояния - это ядерные положительные операторы с единичным следом, а квантовый канал - это произвольное линейное, вполне положительное и сохраняющее след отображение банаховой алгебры ядерных операторов на гильбертовом пространстве, соответствующем входной квантовой системе, в банахову алгебру ядерных операторов на гильбертовом пространстве, соответствующем выходной квантовой системе. Важной характеристикой квантового канала является $\chi$-пропускная способность ${ }^{2)}$. Для канала между конечномерными квантовыми системами $\chi$-пропускная способность всегда конечна и не превосходит максимума выходной энтропии этого канала, которая является непрерывной ограниченной функцией на

* Математический институт им. В. А. Стеклова РАН, ул. Губкина, 8, 119991 Москва, Россия; e-mail: msh@mi.ras.ru

1) Работа выполнена при поддержке научной программы отделения математики РАН «Современные проблемы теоретической математики» и РФФИ (грант № 06-0100164-a).

2) Эта величина, называемая в зарубежной литературе the Holevo capacity, тесно связана с пропускной способностью для передачи классической информации по квантовому каналу связи [3]. 
компактном пространстве входных состояний. Для канала между бесконечномерными квантовыми системами выходная энтропия, как правило, не является ограниченной функцией, что может приводить к бесконечным значениям $\chi$-пропускной способности этого канала. Это обстоятельство приводит к необходимости введения ограничений на множество состояний входной системы, таких, например, как ограничения на среднюю энергию входного ансамбля, которые являются физически мотивированными и обеспечивают конечность $\chi$-пропускной способности [5], [6]. Существует, однако, нетривиальный класс бесконечномерных квантовых каналов, характеризумых конечностью $\chi$-пропускной способности при отсутствии ограничений. Вьходная энтропия таких каналов может быть ограниченной и даже непрерывной, но может принимать и бесконечные значения. Изучению именно этого класса каналов посвящена данная статья.

Из результатов работ [10], [11] следуют некоторые общие свойства каналов с конечной $\chi$-пропускной способностью, такие как относительная компактность выходного множества состояний и существование единственного выходного оптимального среднего состояния (теорема 1). Показано (следствие 1 ), что любой канал с конечной $\chi$-пропускной способностью можно равномерно приблизить последовательностью каналов с конечномерным выходным пространством таким образом, что соответствуюшие последовательности $\chi$-пропускных способностей и выходных оптимальных средних сходятся к $\chi$-пропускной способности и выходному оптимальному среднему этого канала ${ }^{3)}$.

Класс каналов с конечной $\chi$-пропускной способностью содержит каналы с непрерывной выходной энтропией, названные СЕ-каналами. Эти каналы наследуют некоторые аналитические свойства конечномерных каналов, проявляя при этом и свойства, характерные для бесконечномерных каналов. Поэтому класс СЕ-каналов можно рассматривать как промежуточный и использовать технические преимущества работы с этими каналами для исследования бесконечномерньх каналов и систем общего вида. Например, канал этого класса был использован в [2] при изучении свойств бесконечномерных каналов, разрушающих сцепленность, и множества несцепленньх состояний.

В [6] введено понятие оптимальной меры (обобщенного ансамбля) для бесконечномерных каналов с ограничениями и получено достаточное условие ее существования. В данной статье рассмотрены специальные условия существования оптимальной меры для бесконечномерного канала с конечной $\chi$-пропускной способностью (теорема 2 , следствие 2 ) и построены примеры каналов с ограниченной (и даже непрерывной) вы-

3) Последнее утверждение не очевидно, поскольку $\chi$-пропускная способность не является непрерывной функцией канала в топологии равномерной сходимости [17]. 
ходной энтропией, не имеющих оптимальной меры (предложение $3, \mathrm{~B}$ ) и пример 2).

Завершается статья рассмотрением одного класса бесконечномерных каналов, для которых найдена $\chi$-пропускная способность и получено простое условие непрерывности выходной энтропии (предложение 4). CE-каналы этого класса проявляют специфические свойства бесконечномерных каналов такие, как отсутствие входных оптимальных средних состояний и оптимальной меры, разрывность $\chi$-пропускной способности как функции канала (пример 2).

2. Предварительные сведения. Пусть $\mathscr{H}$ - сепарабельное гильбертово пространство, $\mathfrak{B}(\mathscr{H})$ - алгебра всех ограниченных операторов в $\mathscr{H}, \mathfrak{T}(\mathscr{H})$ - банахово пространство всех ядерных операторов со следовой нормой $\|\cdot\|_{1}$. Состоянием далее называется положительный ядерный оператор $\rho$ в $\mathscr{H}$ с единичным следом: $\rho \geqslant 0, \operatorname{Tr} \rho=1$. Алгебра $\mathfrak{B}(\mathscr{H})$ обычно называется алгеброй наблюдаемых квантовой системы, тогда состояние задает функционал математического ожидания $A \mapsto \operatorname{Tr} \rho A ; A \in \mathfrak{B}(\mathscr{H})$, т.е. нормальное состояние в смысле теории операторных алгебр [1]. Множество всех состояний $\mathfrak{S}(\mathscr{H})$ - выпуклое замкнутое подмножество $\mathfrak{T}(\mathscr{H})$, которое является полным сепарабельным метрическим пространством с метрикой, определяемой следовой нормой.

Пусть $A$ и $B$ - положительные операторы из $\mathfrak{T}(\mathscr{H})$. Энтропия фон Неймана оператора $A$ и относительная энтропия операторов $A$ и $B$ определяются соответственно выражениями

$$
\begin{aligned}
H(A) & =-\sum_{i}\langle i|A \log A| i\rangle \\
H(A \| B) & =\sum_{i}\langle i|(A \log A-A \log B+B-A)| i\rangle,
\end{aligned}
$$

в которьх $\{|i\rangle\}$ - базис из собственных векторов оператора $A$ (подробнее см. в [14], [20]). Энтропия и относительная энтропия являются полунепрерывными снизу функциями своих аргументов со значениями в $[0,+\infty]$, первая из которых вогнута, а вторая выпукла по совокупности аргументов [14], [20].

Пусть $\mathscr{H}$ и $\mathscr{H}^{\prime}$ - пара гильбертовых пространств. Канал $\Phi-$ это линейное, положительное, сохраняющее след отображение $\mathfrak{T}(\mathscr{H})$ в $\mathfrak{T}\left(\mathscr{H}^{\prime}\right)$ такое, что двойственное отображение $\Phi^{*}: \mathfrak{B}\left(\mathscr{H}^{\prime}\right) \mapsto \mathfrak{B}(\mathscr{H})$ (которое существует, поскольку $\Phi$ ограничено) является вполне положительным [4]. В частности, канал отображает входные состояния из $\mathfrak{S}(\mathscr{H})$ в выхходные состояния из $\mathfrak{S}\left(\mathscr{H}^{\prime}\right)$.

Для произвольного множества $\mathscr{A}$ обозначим со $(\mathscr{A})$ и $\overline{\text { со }}(\mathscr{A})$ его выпуклую оболочку и его выпуклое замыкание соответственно. Множество всех крайних точек множества $\mathscr{A}$ обозначим $\operatorname{Extr}(\mathscr{A})[8]$. 
Говоря о непрерывности какой-либо функции на некотором множестве состояний, мы будем иметь в виду непрерывность сужения этой функции на данное множество.

Конечный набор состояний $\left\{\rho_{i}\right\}$ с соответствующими вероятностями $\left\{\pi_{i}\right\}$, далее обозначаемый $\left\{\pi_{i}, \rho_{i}\right\}$, называется (конечным) ансамблем, а состояние $\bar{\rho}=\sum_{i} \pi_{i} \rho_{i}-$ средним этого ансамбля. В [6] введено понятие обобщенного ансамбля как произвольной вероятностной борелевской меры $\mu$ на $\mathfrak{S}(\mathscr{H})$. Средним обобщенного ансамбля - меры $\mu$ называется барицентр этой меры, определяемый интегралом Бохнера

$$
\bar{\rho}(\mu)=\int_{\mathfrak{S}(\mathscr{H})} \rho \mu(d \rho) .
$$

Обычное понятие ансамбля соответствует мерам с конечным носителем.

Множество всех вероятностных мер на замкнутом множестве состояний $\mathscr{A}$, снабженное топологией слабой сходимости, обозначим $\mathscr{P}(\mathscr{A})$, а его подмножество, состоящее из мер с конечным носителем, обозначим $\mathscr{P}(\mathscr{A})[19]$. Будем использовать сокращения $\mathscr{P}=\mathscr{P}(\mathfrak{S}(\mathscr{H}))$ и $\mathscr{P}^{\mathrm{f}}=\mathscr{P} \mathbf{f}(\mathfrak{S}(\mathscr{H}))$.

Далее в статье произвольный ансамбль $\left\{\pi_{i}, \rho_{i}\right\}$ будем рассматривать как частный случай вероятностной меры.

Рассмотрим функционалы

$\chi_{\Phi}(\mu)=\int_{\mathfrak{S}(\mathscr{H})} H(\Phi(\rho) \| \Phi(\bar{\rho}(\mu))) \mu(d \rho) \quad$ и $\quad \widehat{H}_{\Phi}(\mu)=\int_{\mathfrak{S}(\mathscr{H})} H(\Phi(\rho)) \mu(d \rho)$.

В [6] показано (предложение 1 и доказательство теоремы), что эти функционалы корректно определены и полунепрерывны снизу на $\mathscr{P}$, причем

$$
\chi_{\Phi}(\mu)=H(\Phi(\bar{\rho}(\mu)))-\widehat{H}_{\Phi}(\mu)
$$

для любой меры $\mu$ такой, что $H(\Phi(\bar{\rho}(\mu)))<+\infty$.

Если $\mu=\left\{\pi_{i}, \rho_{i}\right\}$, то

$$
\chi_{\Phi}\left(\left\{\pi_{i}, \rho_{i}\right\}\right)=\sum_{i} \pi_{i} H\left(\Phi\left(\rho_{i}\right) \| \Phi(\bar{\rho})\right) \quad \text { и } \quad \widehat{H}_{\Phi}\left(\left\{\pi_{i}, \rho_{i}\right\}\right)=\sum_{i} \pi_{i} H\left(\Phi\left(\rho_{i}\right)\right) .
$$

В статье будет использоваться понятие $H$-сходимости последовательности состояний $\left\{\rho_{n}\right\}$ к состоянию $\rho_{0}$, определяемой условием

$$
\lim _{n \rightarrow+\infty} H\left(\rho_{n} \| \rho_{0}\right)=0
$$

и понятие коэффиииента убывания $\mathrm{d}(\sigma)$ состояния $\sigma$, определенного как точная нижняя грань множества $\left\{\lambda>0 \mid \operatorname{Tr} \sigma^{\lambda}<+\infty\right\}$.

В [10] показано (предложение 2), что если $H$ - $\lim _{n \rightarrow+\infty} \rho_{n}=\rho_{0}$, то $\lim _{n \rightarrow+\infty} H\left(\rho_{n}\right)=H\left(\rho_{0}\right)$ при условии $\mathrm{d}\left(\rho_{0}\right)<1$. 
В [10], [11] рассмотрено понятие $\chi$-емкости $\bar{C}(\mathscr{A})$ произвольного множества $\mathscr{A}$ квантовых состояний, определяемой выражением

$$
\bar{C}(\mathscr{A})=\sup _{\left\{\pi_{i}, \rho_{i}\right\} \in \mathscr{P P}^{\mathrm{f}}(\mathscr{A})} \sum_{i} \pi_{i} H\left(\rho_{i} \| \bar{\rho}\right)
$$

(точная верхняя грань в котором берется по множеству всех ансамблей состояний из $\mathscr{A})$, исследованы ее свойства и введено понятие оптимального среднего состояния $\Omega(\mathscr{A})$ множества $\mathscr{A}$ с конечной $\chi$-емкостью. Множество состояний $\mathscr{A}$ с конечной $\chi$-емкостью называется регулярным, если выполнено одно из двух следуюших условий:

1) $H(\Omega(\mathscr{A}))<+\infty$ и $\lim _{n \rightarrow+\infty} H\left(\rho_{n}\right)=H(\Omega(\mathscr{A}))$ для произвольной последовательности $\left\{\rho_{n}\right\}$ состояний из со $(\mathscr{A}), H$-сходяшейся к состоянию $\Omega(\mathscr{A})$;

2) функция $\rho \mapsto H(\rho \| \Omega(\mathscr{A}))$ непрерывна на множестве $\overline{\mathscr{A}}$ (в топологии следовой нормы).

В некотором смысле, условия в данном определении суть минимальные требования непрерывности, обеспечиваюшие «хорошие» свойства $\chi$-емкости (см. теорему 2 в [10] и теорему 1 в [11]). Простейшее достаточное условие регулярности множества $\mathscr{A}$ - неравенство $\mathrm{d}(\Omega(\mathscr{A}))<1$ (утверждение 5 теоремы 1 в [11]), однако это условие не является необходимым и сушествуют регулярные множества, у которых состояние $\Omega(\mathscr{A})$ имеет бесконечную энтропию.

Все обозначения, используемые в статье, согласуются с принятыми в [10], [11].

3. Общие свойства. Пусть $\Phi: \mathfrak{S}(\mathscr{H}) \rightarrow \mathfrak{S}\left(\mathscr{H}^{\prime}\right)-$ канал с конечной $\chi$-пропускной способностью, определяемой выражением

$$
\bar{C}(\Phi)=\sup _{\left\{\pi_{i}, \rho_{i}\right\} \in \mathscr{P} \boldsymbol{f}} \chi_{\Phi}\left(\left\{\pi_{i}, \rho_{i}\right\}\right)=\sup _{\left\{\pi_{i}, \rho_{i}\right\} \in \mathscr{P} \mathrm{r}} \sum_{i} \pi_{i} H\left(\Phi\left(\rho_{i}\right) \| \Phi(\bar{\rho})\right) .
$$

В [6] показано, что

$$
\bar{C}(\Phi)=\sup _{\mu \in \mathscr{P}} \chi_{\Phi}(\mu)=\sup _{\mu \in \mathscr{P}} \int_{\mathfrak{S}(\mathscr{H})} H(\Phi(\rho) \| \Phi(\bar{\rho}(\mu))) \mu(d \rho) .
$$

В соответствии с [9] последовательность ансамблей $\left\{\left\{\pi_{i}^{n}, \rho_{i}^{n}\right\}\right\}_{n}$ такая, что

$$
\lim _{n \rightarrow+\infty} \chi_{\Phi}\left(\left\{\pi_{i}^{n}, \rho_{i}^{n}\right\}\right)=\bar{C}(\Phi)
$$

называется аппроксимирующей последовательностью для канала $\Phi$, а любой частичный предел соответствующей последовательности средних состояний $\left\{\bar{\rho}_{n}=\sum_{i} \pi_{i}^{n} \rho_{i}^{n}\right\}$ называется входныцм оптимальным средним состоянием для канала $\Phi$.

Поскольку для конечномерного канала входное множество состояний компактно, для такого канала всегда существует по крайней мере 
одно входное оптимальное среднее, совпадающее со средним состоянием оптимального ансамбля [18]. Для бесконечномерного канала с конечной $\chi$-пропускной способностью входное оптимальное среднее существует не всегда (пример 1), причем его наличие является необходимым, а при некоторых дополнительных требованиях и достаточным условием существования оптимальной меры для этого канала (теорема 2, следствие 2).

По определению $\chi$-пропускная способность канала $\Phi$ совпадает с $\chi$ емкостью выходного множества $\Phi(\mathfrak{S}(\mathscr{H}))$ этого канала [10]. Поэтому теорема 1 из [10] и теорема 1 из [11] приводят к следующему наблюдению.

Теорема 1. Пусть $\Phi: \mathfrak{S}(\mathscr{H}) \rightarrow \mathfrak{S}\left(\mathscr{H}^{\prime}\right)$ - канал с конечной $\chi-$ пропускной способностью.

1. Множество $\Phi(\mathfrak{S}(\mathscr{H}))$ является относительно компактным подмножеством множества $\mathfrak{S}\left(\mathscr{H}^{\prime}\right)$.

2. Существует единственное состояние $\Omega(\Phi)$ в $\mathfrak{S}\left(\mathscr{H}^{\prime}\right)$ такое, что

$$
H(\Phi(\rho) \| \Omega(\Phi)) \leqslant \bar{C}(\Phi) \quad \forall \rho \in \mathfrak{S}(\mathscr{H}) .
$$

Состояние $\Omega(\Phi)$ лежит в $\overline{\Phi(\mathfrak{S}(\mathscr{H}))}$. Для любой аппроксимирующей последовательности ансамблей $\left\{\left\{\pi_{i}^{n}, \rho_{i}^{n}\right\}\right\}_{n}$ соответствуюшая последовательность $\left\{\Phi\left(\bar{\rho}_{n}\right)\right\}_{n}$ образов их средних состояний $H$-сходится $\kappa$ состоянию $\Omega(\Phi)$.

Если существует входное оптимальное среднее состояние $\rho_{*}$ для канала $\Phi$, то $\Phi\left(\rho_{*}\right)=\Omega(\Phi)$. нue $e^{4)}$

3. Для $\chi$-пропускной способности канала $\Phi$ имеет место выраже-

$$
\bar{C}(\Phi)=\inf _{\sigma \in \mathfrak{S}\left(\mathscr{H}{ }^{\prime}\right)} \sup _{\rho \in \mathfrak{S}(\mathscr{H})} H(\Phi(\rho) \| \sigma)=\sup _{\rho \in \mathfrak{S}(\mathscr{H})} H(\Phi(\rho) \| \Omega(\Phi))
$$

В соответствии с [9] состояние $\Omega(\Phi)$ называется выходным оптимальным средним состоянием для канала $\Phi$.

Ниже мы рассмотрим примеры каналов с конечной $\chi$-пропускной способностью, не имеющих входных оптимальных средних состояний, для которых выходное оптимальное среднее состояние явно определяется и играет важную роль в изучении этих каналов.

Для конечномерного канала $\Phi$ состояние $\Omega(\Phi)$ - это образ среднего состояния любого оптимального ансамбля для этого канала и поэтому

$$
\Omega(\Phi) \in \Phi(\mathfrak{S}(\mathscr{H})) \quad \text { и } \quad \bar{C}(\Phi) \leqslant H(\Omega(\Phi)) .
$$

4) В силу следствия 5 из [11] точную нижнюю грань в этом выражении можно брать по подмножеству множества $\overline{\Phi(\mathfrak{S}(\mathscr{H}))}$, состоящему из состояний, инвариантных относительно всех симметрий $\alpha$ пространства $\mathfrak{S}\left(\mathscr{H}^{\prime}\right)$ таких, что $\alpha(\overline{\Phi(\mathfrak{S}(\mathscr{H}))}) \subseteq$ $\overline{\Phi(\mathfrak{S}(\mathscr{H}))}$. 
Для бесконечномерного канала с конечной $\chi$-пропускной способностью данные соотношения в общем случае не имеют места (см. предложение $3, \mathrm{~B})$ ). Включение в (4) следует из существования по крайней мере одного входного оптимального среднего для канала $\Phi$, а достаточное условие справедливости неравенства в (4) можно выразить в терминах характеристики спектра состояния $\Omega(\Phi)$ (см. предложение 2).

Простейшие бесконечномерные каналы с конечной $\chi$-пропускной способностью - это каналы из бесконечномерной квантовой системы в конечномерную. Следуя [17], такие каналы будем называть IF-каналами. Из теоремы 1 следует, в частности, что каждый канал с конечной $\chi$-пропускной способностью можно равномерно приблизить последовательностью IF-каналов.

Следствие 1. Канал $\Phi: \mathfrak{S}(\mathscr{H}) \rightarrow \mathfrak{S}\left(\mathscr{H}^{\prime}\right)$ имеет конечную $\chi-$ пропускную способность тогда и только тогда, когда существует последовательность IF-каналов $\left\{\Phi_{n}: \mathfrak{S}(\mathscr{H}) \rightarrow \mathfrak{S}\left(\mathscr{H}_{n}^{\prime}\right), \mathscr{H}_{n}^{\prime} \subseteq \mathscr{H}^{\prime}\right\}$ такая, umo

$$
\lim _{n \rightarrow+\infty} \sup _{\rho \in \mathfrak{S}(\mathscr{H})}\left\|\Phi_{n}(\rho)-\Phi(\rho)\right\|_{1}=0 \quad u \quad \sup _{n} \bar{C}\left(\Phi_{n}\right)<+\infty
$$

Последовательность $\left\{\Phi_{n}\right\}$ можно выбрать таким образом, что

$$
\lim _{n \rightarrow+\infty} \bar{C}\left(\Phi_{n}\right)=\bar{C}(\Phi) \quad u \quad \lim _{n \rightarrow+\infty} \Omega\left(\Phi_{n}\right)=\Omega(\Phi)
$$

Д о к а з а т е л ь с т в о. Если указанная последовательность $\left\{\Phi_{n}\right\}$ существует, то из полунепрерывности снизу $\chi$-пропускной способности как функции канала, установленной в [17], следует, что $\chi$-пропускная способность канала $\Phi$ конечна.

Если $\chi$-пропускная способность канала $\Phi$ конечна, то, в силу теоремы 1, множество $\Phi(\mathfrak{S}(\mathscr{H}))$ относительно компактно. Пусть $\left\{P_{n}\right\}-$ последовательность проекторов конечного ранга в $\mathscr{H}^{\prime}$, сильно сходящаяся к единичному оператору $I_{\mathscr{H}}$. Из критерия компактности (см. приложение в [6]) следует, что $\lim _{n \rightarrow+\infty} \inf _{\rho \in \mathfrak{S}(\mathscr{H})} \operatorname{Tr} \Phi(\rho) P_{n}=1$. Поэтому последовательность IF-каналов

$$
\Phi_{n}(\rho)=P_{n} \Phi(\rho) P_{n}+\left(\operatorname{Tr}\left(I_{\mathscr{H}},-P_{n}\right) \Phi(\rho)\right) \tau_{n}
$$

из $\mathfrak{S}(\mathscr{H})$ в $\mathfrak{S}\left(P_{n}\left(\mathscr{H}^{\prime}\right) \oplus \mathscr{H}_{n}^{\prime \prime}\right)$, где $\tau_{n}$ - чистое состояние, соответствующее произвольному единичному вектору из некоторого конечномерного подпространства $\mathscr{H}_{n}^{\prime \prime} \subseteq \mathscr{H}^{\prime} \ominus P_{n}\left(\mathscr{H}^{\prime}\right)$, равномерно сходится к каналу $\Phi$. Поскольку при каждом $n$ канал $\Phi_{n}$ можно представить в виде композиции канала $\Phi$ и канала $\rho \mapsto P_{n} \rho P_{n}+\left(\operatorname{Tr}\left(I_{\mathscr{H}},-P_{n}\right) \rho\right) \tau_{n}$, из свойства монотонности относительной энтропии (см. [15], [20]) вытекает неравенство $\bar{C}\left(\Phi_{n}\right) \leqslant \bar{C}(\Phi)$ для всех $n$. С учетом этого неравенства предельные 
выражения во второй части следствия доказываются с помошью леммы 1 из [11].

Следствие 1 показывает, что класс каналов с конечной $\chi$-пропускной способностью достаточно близок к классу IF-каналов. Тем не менее, каналы этого класса проявляют многие специфические свойства бесконечномерных каналов (см. примеры 1 и 2).

Существуют бесконечномерные каналы с конечной $\chi$-пропускной способностью, которые наиболее близки по своим аналитическим свойствам к конечномерным каналам.

О п р е д е л е н и е 1 . Канал $\Phi: \mathfrak{S}(\mathscr{H}) \rightarrow \mathfrak{S}\left(\mathscr{H}^{\prime}\right)$ называется СЕканалом, если сужение квантовой энтропии на множество $\overline{\Phi(\mathfrak{S}(\mathscr{H}))}$ является непрерывным (в топологии следовой нормы).

Используя результаты из [10], [11], можно получить различные достаточные условия СЕ-свойства (см. предложение 2). В частности, из $[11$, предложение 8] следует, что класс СЕ-каналов замкнут относительно тензорных произведений: если $\Phi: \mathfrak{S}(\mathscr{H}) \rightarrow \mathfrak{S}\left(\mathscr{H}^{\prime}\right) u \Psi: \mathfrak{S}(\mathscr{K}) \rightarrow$ $\mathfrak{S}\left(\mathscr{K}^{\prime}\right)$ CE-каналь, то канал $\Phi \otimes \Psi: \mathfrak{S}(\mathscr{H} \otimes \mathscr{K}) \rightarrow \mathfrak{S}\left(\mathscr{H}^{\prime} \otimes \mathscr{K}^{\prime}\right)$ также является СЕ-каналом.

$\chi$-пропускная способность канала $\Phi$ совпадает с точной верхней гранью $\chi$-функции канала $\Phi$, определяемой выражением

$$
\begin{aligned}
\chi_{\Phi}(\rho) & =\sup _{\left\{\pi_{i}, \rho_{i}\right\} \in \mathscr{P}_{\{\mathfrak{f}\}} \sum_{\{\rho\}} \pi_{i} H\left(\Phi\left(\rho_{i}\right) \| \Phi(\rho)\right)} \\
& =\sup _{\mu \in \mathscr{P}_{\{\rho\}}} \int_{\mathfrak{S}(\mathscr{H})} H(\Phi(\sigma) \| \Phi(\rho)) \mu(d \sigma),
\end{aligned}
$$

где $\mathscr{P}_{\{\rho\}}-$ множество всех вероятностных мер на $\mathfrak{S}(\mathscr{H})$ с барицентром $\rho$ (см. [6]).

В [9] показано, что выпуклое замыкание выходной энтропии канала $\Phi$ определяется выражением

$$
\widehat{H}_{\Phi}(\rho)=\inf _{\mu \in \mathscr{P}} \int_{\{\rho\}} H(\Phi(\rho)) \mu(d \rho) \leqslant+\infty .
$$

В отличие от $\chi$-функции, в определении $\widehat{H}$-функции точная нижняя грань по всем мерам из $\mathscr{P}_{\{\rho\}}$, вообще говоря, не совпадает с точной нижней гранью по всем мерам из $\mathscr{P}_{\{\rho\}}$ с конечным носителем (лемма 2 в [9]).

$\chi$-функция и $\widehat{H}$-функция произвольного канала - неотрицательные полунепрерывные снизу вогнутая и выпуклая функции соответственно [9]. Следующее предложение показывает, в частности, что свойства $\chi$-функции и $\widehat{H}$-функции СЕ-канала близки к свойствам соответствующих функций конечномерного канала. 
Предложение 1. Пусть $\Phi-$ канал с конечной $\chi$-пропускной способностью. Тогда

$$
\chi_{\Phi}(\rho) \leqslant \bar{C}(\Phi)-H(\Phi(\rho) \| \Omega(\Phi)) \quad \forall \rho \in \mathfrak{S}(\mathscr{H}) .
$$

Если $\Phi$ есть СЕ-канал, то функиии $\chi_{\Phi}$ и $\widehat{H}_{\Phi}$ непрерывны и ограничены на $\mathfrak{S}(\mathscr{H})$, причем для любого $\rho \in \mathfrak{S}(\mathscr{H})$

$$
\chi_{\Phi}(\rho)=H(\Phi(\rho))-\widehat{H}_{\Phi}(\rho) \quad u \quad \widehat{H}_{\Phi}(\rho)=\inf _{\left\{\pi_{i}, \rho_{i}\right\} \in \mathscr{P}_{(\rho)}} \sum_{i} \pi_{i} H\left(\Phi\left(\rho_{i}\right)\right) .
$$

Д о к а з а т е л ь с т в о. Неравенство для $\chi$-функции вытекает из предложения 3 в [9]. Утверждение о непрерывности и представления для $\chi$-функции и для $\widehat{H}$-функции следуют из предложения 5 и теоремы 1 в [9].

Следующее предложение показывает особую роль выходного оптимального среднего состояния $\Omega(\Phi)$.

Предложение 2. Пусть $\Phi-$ канал с конечной $\chi$-пропускной способностью.

$E c \wedge u \mathrm{~d}(\Omega(\Phi))<1$, mo $\sup _{\rho \in \mathfrak{S}(\mathscr{H})} H(\Phi(\rho))<+\infty u \bar{C}(\Phi) \leqslant H(\Omega(\Phi))$.

Eсли $\mathrm{d}(\Omega(\Phi))=0$, то $\Phi$ является СЕ-каналом.

Д ок а з а т ел в с т в о. Предположим, что $\mathrm{d}(\Omega(\Phi))<1$. Из утверждения 5 теоремы 1 в [11] следует, что энтропия ограничена на множестве $\overline{\Phi(\mathfrak{S}(\mathscr{H}))}$. В силу теоремы 1 для произвольной аппроксимируюшей последовательности ансамблей $\left\{\left\{\pi_{i}^{n}, \rho_{i}^{n}\right\}\right\}_{n}$ соответствующая последовательность $\left\{\Phi\left(\bar{\rho}_{n}\right)\right\}_{n}$ образов средних состояний $H$-сходится к состоянию $\Omega(\Phi)$. В силу предложения 2 в [10] из условия $\mathrm{d}(\Omega(\Phi))<1$ следует, что

$$
\bar{C}(\Phi)=\lim _{n \rightarrow+\infty} \chi_{\Phi}\left(\left\{\pi_{i}^{n}, \rho_{i}^{n}\right\}\right) \leqslant \lim _{n \rightarrow+\infty} H\left(\Phi\left(\bar{\rho}_{n}\right)\right)=H(\Omega(\Phi)) .
$$

Предположим, что $\mathrm{d}(\Omega(\Phi))=0$. Из утверждения 5 теоремы 1 в [11] следует, что энтропия непрерывна на множестве $\overline{\Phi(\mathfrak{S}(\mathscr{H}))}$.

Утверждения данного предложения иллюстрируются в приведенном ниже примере 1 , в котором рассмотрено семейство каналов с различными коэффициентами убывания выходного оптимального среднего состояния.

3 а м е ч а н и е 1 . Пусть $\widehat{\mathfrak{S}}(\mathscr{H})-$ подмножество пространства $(\mathfrak{B}(\mathscr{H}))^{*}$, состоящее из положительньг функционалов на $\mathfrak{B}(\mathscr{H})$ с единичной нормой. Известно, что $\widehat{\mathfrak{S}}(\mathscr{H})$ компактно в $*$-слабой топологии и что $\mathfrak{S}(\mathscr{H})$ является $*$-слабо плотным подмножеством множества $\widehat{\mathfrak{S}}(\mathscr{H})$ [1]. Предкомпактность множества значений произвольного канала $\Phi: \mathfrak{S}(\mathscr{H}) \rightarrow \mathfrak{S}\left(\mathscr{H}^{\prime}\right)$ с конечной $\chi$-пропускной способностью, гарантируемая теоремой 1 , обеспечивает существование у этого канала расширения на $\widehat{\mathfrak{S}}(\mathscr{H})$, т.е. такого отображения $\widehat{\Phi}: \widehat{\mathfrak{S}}(\mathscr{H}) \rightarrow \mathfrak{S}\left(\mathscr{H}^{\prime}\right)$, непрерывного относительно $*$-слабой топологии на множестве $\widehat{\mathfrak{S}}(\mathscr{H})$ и топологии следовой нормы на множестве $\mathfrak{S}\left(\mathscr{H}^{\prime}\right)$, что $\left.\widehat{\Phi}\right|_{\mathfrak{S}(\mathscr{H})}=\Phi$. Можно показать, что расширение канала не приводит к увеличению $\chi$-пропускной 
способности, но может приводить к уменьшению минимальной выходной энтропии.

4. Оптимальная мера. В соответствии с [6] мера $\mu_{*}$ из $\mathscr{P}(\operatorname{Extr} \mathfrak{S}(\mathscr{H}))$, на которой достигается точная верхняя грань в определении (3), называется оптимальной мерой (обобщенным оптимальным ансамблем) для канала $\Phi$.

Понятие оптимальной меры является обобщением понятия оптимального ансамбля для конечномерного канала. В [18] показано, что каждый оптимальный ансамбль характеризуется свойством максимальной равноудаленности. В первой части следующей теоремы это свойство обобщается на случай бесконечномерных каналов.

Теорема 2. Пусть $\Phi: \mathfrak{S}(\mathscr{H}) \rightarrow \mathfrak{S}\left(\mathscr{H}^{\prime}\right)-$ канал с конечной $\chi$-пропускной способностью.

Если сушествует оптимальная мера $\mu_{*}$ для канала $\Phi$, то ее баричентр $\bar{\rho}\left(\mu_{*}\right)$ является входньм оптимальным средним состоянием для канала $\Phi$ и имеет место следующее свойство максимальной равноудаленности:

$$
H(\Phi(\rho) \| \Omega(\Phi))=\bar{C}(\Phi) \quad \text { для } \mu_{*} \text {-nочти всех } \rho \text { из } \mathfrak{S}(\mathscr{H})
$$

Если существует входное оптимальное среднее состояние $\rho_{*}$ для канала $\Phi$ и множество $\Phi(\mathfrak{S}(\mathscr{H}))$ является регулярным ${ }^{5)}$, то существует оптимальная мера $\mu_{*}$ для канала $\Phi$ такая, что $\bar{\rho}\left(\mu_{*}\right)=\rho_{*}$.

Д о к а з а т л ь с в о. Поскольку каждую вероятностную меру можно аппроксимировать слабо сходящейся последовательностью мер с конечным носителем, из полунепрерывности снизу функционала $\chi_{\Phi}$ следует, что барицентр $\bar{\rho}\left(\mu_{*}\right)$ любой оптимальной меры $\mu_{*}$ для канала $\Phi$ является входным оптимальным средним состоянием для этого канала. Свойство максимальной равноудаленности следует из определения оптимальной меры и теоремы 1.

Предположим, что множество $\Phi(\mathfrak{S}(\mathscr{H}))$ является регулярным и $\left\{\mu_{n}=\left\{\pi_{i}^{n}, \rho_{i}^{n}\right\}\right\}_{n}$ - аппроксимирующая последовательность ансамблей для канала $\Phi$ такая, что соответствующая последовательность $\left\{\bar{\rho}\left(\mu_{n}\right)\right\}_{n}$ сходится к состоянию $\rho_{*}$. В силу выпуклости и полунепрерывности снизу относительной энтропии можно считать, что каждый ансамбль в этой последовательности состоит из чистых состояний. Поскольку последовательность $\left\{\bar{\rho}\left(\mu_{n}\right)\right\}_{n}$ предкомпактна, последовательность $\left\{\mu_{n}\right\}_{n}$ слабо предкомпактна в силу предложения 2 в [6] и, следовательно, она содержит слабо сходящуюся подпоследовательность. Поэтому без ограничения общности можно считать, что последовательность $\left\{\mu_{n}\right\}_{n}$

5) См. определение в п. 2 (с. 736$)$. 
слабо сходится к некоторой мере $\mu_{*}$, носитель которой, в силу теоремы 6.1 в [19], состоит из чистьг состояний.

Для каждого $n$ пусть $\nu_{n}=\mu_{n} \circ \Phi^{-1}$ - образ меры $\mu_{n}$ при отображении $\Phi$, так что $\nu_{n}=\left\{\pi_{i}^{n}, \Phi\left(\rho_{i}^{n}\right)\right\}$. Нетрудно видеть, что $\left\{\nu_{n}\right\}_{n}$ - аппроксимирующая последовательность ансамблей для множества $\overline{\Phi(\mathfrak{S}(\mathscr{H}))}$ в терминологии [10]. Поскольку это множество регулярно по условию, рассуждения в доказательстве теоремы 2 в [10] показывают существование подпоследовательности мер $\left\{\nu_{n_{k}}\right\}_{k}$, слабо сходящейся к оптимальной мере $\nu_{*}$ для множества $\overline{\Phi(\mathfrak{S}(\mathscr{H}))}$. Но из слабой сходимости последовательности $\left\{\mu_{n_{k}}\right\}_{k}$ к мере $\mu_{*}$ следует слабая сходимость последовательности $\left\{\mu_{n_{k}} \circ \Phi^{-1}\right\}_{k}$ к мере $\mu_{*} \circ \Phi^{-1}$. Поэтому $\mu_{*} \circ \Phi^{-1}=\nu_{*}$, а значит, $\mu_{*}-$ оптимальная мера для канала $\Phi$. Теорема 2 доказана.

Утверждение 5 теоремы 1 в [11] и теорема 2 приводят к следуюшему достаточному условию существования оптимальной меры.

Следствие 2. Если существует входное оптимальное среднее состояние для канала $\Phi$ и выполнено одно из следующих условий:

1) $\mathrm{d}(\Omega(\Phi))<1$

2) $\Phi$ есть СЕ-канал, то существует оптимальная мера для канала $\Phi$.

Следствие 2 может быть также использовано для доказательства отсутствия входных оптимальных средних состояний у таких каналов, для которых легко доказывается отсутствие оптимальной меры (см. пример 2 в п. 5).

3 а мечан и е 2. Условия в теореме 2 и в следствии 2 являются существенными, как показывают примеры каналов с конечной $\chi$ пропускной способностью, для которых не существует оптимальных мер (предложение 3, В) и пример 2).

Для иллюстрации приведенных выше обших результатов рассмотрим следующее семейство каналов, зависяших от последовательности положительных чисел.

П р и м е р 1. Пусть $\{|n\rangle\}_{n \in \mathrm{N} \cup\{0\}}$ - ортонормированный базис в пространстве $\mathscr{H}^{\prime}$ и $\left\{q_{n}\right\}_{n \in \mathbf{N}}$ - последовательность чисел из $(0,1]$, сходящаяся к нулю. Рассмотрим множество $\mathscr{S}_{\left\{q_{n}\right\}}^{1}$, состоящее из чистых состояний

$\sigma_{k}=\left(1-q_{|k|}\right)|0\rangle\left\langle 0\left|+q_{|k|}\right||k|\right\rangle\langle| k \|+\operatorname{sign}(k) \sqrt{\left(1-q_{|k|}\right) q_{|k|}}(|0\rangle\langle|k\|+\| k|\rangle\langle 0|)$,

индексируемых множеством $\mathbf{Z} \backslash\{0\}$. Множество состояний $\mathscr{S}_{\left\{q_{n}\right\}}^{1}$ можно рассматривать как последовательность, сходящуюся к состоянию $|0\rangle\langle 0|$. Свойства этого множества исследованы в примере 1 подраздела 3.1 в [11].

Рассмотрим канал

$$
\Phi_{\left\{q_{n}\right\}}(\rho)=\sum_{k \in \mathbf{Z} \backslash\{0\}}\langle k|\rho| k\rangle \sigma_{k},
$$


где $\{|k\rangle\}_{k \in \mathbf{Z} \backslash\{0\}}$ - некоторый ортонормированный базис в $\mathscr{H}$.

Канал $\Phi_{\left\{q_{n}\right\}}$ является каналом, разрушаюшим сцепленность (см. [16], [2]), причем $\overline{\Phi_{\left\{q_{n}\right\}}(\mathfrak{S}(\mathscr{H}))}=\overline{c o}\left(\mathscr{S}_{\left\{q_{n}\right\}}^{1}\right)$ - компактное подмножество $\mathfrak{S}\left(\mathscr{H}^{\prime}\right)$. Поскольку последовательность $\mathscr{S}_{\left\{q_{n}\right\}}^{1}$ состоит из чистых состояний, нетрудно видеть, что $\bar{C}\left(\Phi_{\left\{q_{n}\right\}}\right)=\sup _{\rho \in \mathcal{S}(\mathscr{H})} H\left(\Phi_{\left\{q_{n}\right\}}(\rho)\right)$. Пусть $\lambda_{\left\{q_{n}\right\}}^{*}-$ точная нижняя грань множества $\left\{\lambda: \sum_{n} \exp \left(-\lambda / q_{n}\right)<+\infty\right\}$, если оно не пусто, и $\lambda_{\left\{q_{n}\right\}}^{*}=+\infty$ в противном случае. Пусть

$$
F_{\left\{q_{n}\right\}}(\lambda)=\sum_{n=1}^{+\infty}\left(\frac{1}{q_{n}}-1\right) \exp \left(-\frac{\lambda}{q_{n}}\right)
$$

- убывающая функция на $\left[\lambda_{\left\{q_{n}\right\}}^{*},+\infty\right)$ с множеством значений $(0,+\infty]$.

Предложение 3. Справедливы следуюшие утверждения.

A) $\operatorname{Ecлu} \lambda_{\left\{q_{n}\right\}}^{*}=0$, mo

- $\Phi_{\left\{q_{n}\right\}}$ является СЕ-каналом;

- существуют единственная оптимальная мера и единственное входное оптимальное среднее для канала $\Phi_{\left\{q_{n}\right\}}$;

$-\Omega\left(\Phi_{\left\{q_{n}\right\}}\right) \in \Phi_{\left\{q_{n}\right\}}(\mathfrak{S}(\mathscr{H})), \mathrm{d}\left(\Omega\left(\Phi_{\left\{q_{n}\right\}}\right)\right)=0 u \bar{C}\left(\Phi_{\left\{q_{n}\right\}}\right)=$ $H\left(\Omega\left(\Phi_{\left\{q_{n}\right\}}\right)\right)$.

Б)

- $\bar{C}\left(\Phi_{\left\{q_{n}\right\}}\right)<+\infty$, но $\Phi_{\left\{q_{n}\right\}}$ не является CЕ-каналом;

- существуют единственная оптимальная мера и единственное входное оптимальное среднее для канала $\Phi_{\left\{q_{n}\right\}}$;

$-\Omega\left(\Phi_{\left\{q_{n}\right\}}\right) \in \Phi_{\left\{q_{n}\right\}}(\mathfrak{S}(\mathscr{H})) ; 0<\mathrm{d}\left(\Omega\left(\Phi_{\left\{q_{n}\right\}}\right)\right) \leqslant 1 u \bar{C}\left(\Phi_{\left\{q_{n}\right\}}\right)=$ $\left.H\left(\Omega\left(\Phi_{\left\{q_{n}\right\}}\right)\right) .{ }^{6}\right)$

B) $E \operatorname{cлu} 0<\lambda_{\left\{q_{n}\right\}}^{*}<+\infty u F_{\left\{q_{n}\right\}}\left(\lambda_{\left\{q_{n}\right\}}^{*}\right)<1$, mo

- $\bar{C}\left(\Phi_{\left\{q_{n}\right\}}\right)<+\infty$, но $\Phi_{\left\{q_{n}\right\}}$ не является СЕ-каналом;

- не существует ни оптимальной меры, ни входного оптимального среднего для канала $\Phi_{\left\{q_{n}\right\}}$;

$-\underline{\Omega}\left(\Phi_{\left\{q_{n}\right\}}\right) \in \overline{\Phi_{\left\{q_{n}\right\}}(\mathfrak{S}(\mathscr{H}))} \backslash \Phi_{\left\{q_{n}\right\}}(\mathfrak{S}(\mathscr{H})), \mathrm{d}\left(\Omega\left(\Phi_{\left\{q_{n}\right\}}\right)\right)=1 u$ $\bar{C}\left(\Phi_{\left\{q_{n}\right\}}\right)>H\left(\Omega\left(\Phi_{\left\{q_{n}\right\}}\right)\right)$.

Г) $E \operatorname{csu} \lambda_{\left\{q_{n}\right\}}^{*}=+\infty, \operatorname{mo} \bar{C}\left(\Phi_{\left\{q_{n}\right\}}\right)=+\infty$

6) В этом случае $\mathrm{d}\left(\Omega\left(\Phi_{\left\{q_{n}\right\}}\right)\right)=1$ тогда и только тогда, когда $F_{\left\{q_{n}\right\}}\left(\lambda_{\left\{q_{n}\right\}}^{*}\right)=1$. 
В случаях A) $и$ Б) $\chi$-пропускная способность, единственная оптимальная мера, единственное входное и выходное оптимальные средние для канала $\Phi_{\left\{q_{n}\right\}}$ определяются соответственно выражениями:

$$
\begin{aligned}
\bar{C}\left(\Phi_{\left\{q_{n}\right\}}\right) & =\lambda_{\left\{q_{n}\right\}}^{1}-\log \pi_{\left\{q_{n}\right\}}^{1}, \quad \mu_{*}=\left\{\frac{\pi_{\left\{q_{n}\right\}}^{1}}{2 q_{|k|}} \exp \left(-\frac{\lambda_{\left\{q_{n}\right\}}^{1}}{q_{|k|}}\right),|k\rangle\langle k|\right\}_{k \in \mathbf{Z} \backslash\{0\}}, \\
\bar{\rho}\left(\mu_{*}\right) & =\sum_{k \in \mathbf{Z} \backslash\{0\}} \frac{\pi_{\left\{q_{n}\right\}}^{1}}{2 q_{|k|}} \exp \left(-\frac{\lambda_{\left\{q_{n}\right\}}^{1}}{q_{|k|}}\right)|k\rangle\langle k|, \\
\Omega\left(\Phi_{\left\{q_{n}\right\}}\right) & =\pi_{\left\{q_{n}\right\}}^{1}|0\rangle\left\langle 0\left|+\pi_{\left\{q_{n}\right\}}^{1} \sum_{n=1}^{+\infty} \exp \left(-\frac{\lambda_{\left\{q_{n}\right\}}^{1}}{q_{n}}\right)\right| n\right\rangle\langle n|,
\end{aligned}
$$

где $\lambda_{\left\{q_{n}\right\}}^{1}$ - единственное решение уравнения $F_{\left\{q_{n}\right\}}(\lambda)=1 u$

$\pi_{\left\{q_{n}\right\}}^{1}=\left(1+\sum_{n=1}^{+\infty} \exp \left(-\frac{\lambda_{\left\{q_{n}\right\}}^{1}}{q_{n}}\right)\right)^{-1}=\left(\sum_{n=1}^{+\infty} \frac{1}{q_{n}} \exp \left(-\frac{\lambda_{\left\{q_{n}\right\}}^{1}}{q_{n}}\right)\right)^{-1} \in[0,1]$.

В случае В) $\chi$-пропускная способность и выходное оптимальное среднее для канала $\Phi_{\left\{q_{n}\right\}}$ определяются соответственно выражениями:

$$
\begin{aligned}
& \bar{C}\left(\Phi_{\left\{q_{n}\right\}}\right)=\lambda_{\left\{q_{n}\right\}}^{*}-\log \pi_{\left\{q_{n}\right\}}^{*}, \\
& \Omega\left(\Phi_{\left\{q_{n}\right\}}\right)=\pi_{\left\{q_{n}\right\}}^{*}|0\rangle\left\langle 0\left|+\pi_{\left\{q_{n}\right\}}^{*} \sum_{n=1}^{+\infty} \exp \left(-\frac{\lambda_{\left\{q_{n}\right\}}^{*}}{q_{n}}\right)\right| n\right\rangle\langle n|,
\end{aligned}
$$

2дe

$$
\pi_{\left\{q_{n}\right\}}^{*}=\left(1+\sum_{n=1}^{+\infty} \exp \left(-\frac{\lambda_{\left\{q_{n}\right\}}^{*}}{q_{n}}\right)\right)^{-1} \in[0,1] .
$$

Доказательство. Если $\lambda_{\left\{q_{n}\right\}}^{*}=+\infty$, то $\bar{C}\left(\Phi_{\left\{q_{n}\right\}}\right)=$ $\bar{C}\left(\mathscr{S}_{\left\{q_{n}\right\}}^{1}\right)=+\infty$ в силу предложения 4 в [11].

Пусть $\lambda_{\left\{q_{n}\right\}}^{*}<+\infty$. Без ограничения обшности можно считать, что $q_{n+1} \leqslant q_{n}$ для всех $n$. Пусть

$$
H=0|0\rangle\left\langle 0\left|+\sum_{n=1}^{+\infty} q_{n}^{-1}\right| n\right\rangle\langle n|
$$

- $\mathfrak{H}$-оператор в $\mathscr{H}^{\prime}$ такой, что $\mathrm{g}(H)=\lambda_{\left\{q_{n}\right\}}^{*}$ (см. $[10, \S 3]$ ). Нетрудно видеть, что $\mathscr{S}_{\left\{q_{n}\right\}}^{1} \subseteq \mathscr{K}_{H, 1}$ и поэтому из предложения 6 в [11] следует, что $\bar{C}\left(\Phi_{\left\{q_{n}\right\}}\right)=\bar{C}\left(\mathscr{S}_{\left\{q_{n}\right\}}^{1}\right) \leqslant \bar{C}\left(\mathscr{K}_{H, 1}\right)=\sup _{\rho \in \mathscr{K}_{H, 1}} H(\rho)<+\infty$. Покажем, что $\bar{C}\left(\mathscr{S}_{\left\{q_{n}\right\}}^{1}\right)=\bar{C}\left(\mathscr{K}_{H, 1}\right)$. В доказательстве предложения 1 в [10] (см. формулу (8)) построена последовательность $\left\{\rho_{n}\right\}$ состояний и показано, что $\lim _{n \rightarrow+\infty} H\left(\rho_{n}\right)=\sup _{\rho \in \mathscr{K}_{H, 1}} H(\rho)$. Для $\mathfrak{H}$-оператора $H$, определенного 
формулой (6), состояния данной последовательности имеют вид

$$
\rho_{n}=\left(1+\sum_{k=1}^{n} \exp \left(-\frac{\lambda_{n}}{q_{k}}\right)\right)^{-1}\left(|0\rangle\left\langle 0\left|+\sum_{k=1}^{n} \exp \left(-\frac{\lambda_{n}}{q_{k}}\right)\right| k\right\rangle\langle k|\right)
$$

для достаточно больших $n \in \mathbf{N}$, где $\lambda_{n}$ - единственное решение уравнения

$$
1+\sum_{k=1}^{n} \exp \left(-\frac{\lambda}{q_{k}}\right)=\sum_{k=1}^{n} \frac{1}{q_{k}} \exp \left(-\frac{\lambda}{q_{k}}\right) .
$$

Последовательность состояний, определенных формулой (7), лежит в $\overline{\mathrm{co}}\left(\mathscr{S}_{\left\{q_{n}\right\}}^{1}\right)$. Действительно, для данного $n$ рассмотрим распределение вероятностей

$$
\pi_{k}^{n}=\left(\sum_{j=1}^{n} \frac{2}{q_{j}} \exp \left(-\frac{\lambda_{n}}{q_{j}}\right)\right)^{-1} \frac{1}{q_{|k|}} \exp \left(-\frac{\lambda_{n}}{q_{|k|}}\right), \quad 0<|k| \leqslant n .
$$

Учитывая равенство (8) при $\lambda=\lambda_{n}$, нетрудно видеть, что $\sum_{k} \pi_{k}^{n} \sigma_{k}=\rho_{n}$ и, следовательно, $\rho_{n} \in \overline{\text { со }}\left(\mathscr{S}_{\left\{q_{n}\right\}}^{1}\right)$. Поэтому из предложения 6 работы [11] и утверждения 3 теоремы 1 этой же работы следует, что

$$
\bar{C}\left(\Phi_{\left\{q_{n}\right\}}\right)=\bar{C}\left(\mathscr{S}_{\left\{q_{n}\right\}}^{1}\right)=\bar{C}\left(\mathscr{K}_{H, 1}\right)=\lambda^{*}-\log \pi^{*}
$$

и

$$
\Omega\left(\Phi_{\left\{q_{n}\right\}}\right)=\Omega\left(\mathscr{S}_{\left\{q_{n}\right\}}^{1}\right)=\Omega\left(\mathscr{K}_{H, 1}\right)=\pi^{*}|0\rangle\left\langle 0\left|+\pi^{*} \sum_{n=1}^{+\infty} \exp \left(-\frac{\lambda^{*}}{q_{n}}\right)\right| n\right\rangle\langle n|,
$$

где $\pi^{*}=\left(1+\sum_{n=1}^{+\infty} \exp \left(-\lambda^{*} / q_{n}\right)\right)^{-1}$ и $\lambda^{*}$ - единственное решение уравнения

$$
1+\sum_{k=1}^{+\infty} \exp \left(-\frac{\lambda}{q_{k}}\right)=\sum_{k=1}^{+\infty} \frac{1}{q_{k}} \exp \left(-\frac{\lambda}{q_{k}}\right)
$$

если

$$
h_{*}(H)=\frac{\operatorname{Tr} H \exp (-\mathrm{g}(H) H)}{\operatorname{Tr} \exp (-\mathrm{g}(H) H)}=\frac{\sum_{k=1}^{+\infty}\left(1 / q_{k}\right) \exp \left(-\lambda_{\left\{q_{n}\right\}}^{*} / q_{k}\right)}{1+\sum_{k=1}^{+\infty} \exp \left(-\lambda_{\left\{q_{n}\right\}}^{*} / q_{k}\right)} \geqslant h=1,
$$

и $\lambda^{*}=\lambda_{\left\{q_{n}\right\}}^{*}$ в противном случае. Нетрудно видеть, что (11) равносильно уравнению $F_{\left\{q_{n}\right\}}(\lambda)=1$, а $(12)$ - неравенству $F_{\left\{q_{n}\right\}}\left(\lambda_{\left\{q_{n}\right\}}^{*}\right) \geqslant 1$.

Утверждение о существовании и единственности оптимальной меры $\mu_{*}$ для канала $\Phi_{\left\{q_{n}\right\}}$ в случаях A) и Б), а также выражение для этой меры следуют из предложения 4 в [11] (с $\varepsilon=1)$. Барицентр меры $\mu_{*}-$ единственное входное оптимальное среднее для канала $\Phi_{\left\{q_{n}\right\}}$. Единственность этого среднего вытекает из единственности оптимальной меры, поскольку предложение 6 в [11] с учетом (10) гарантирует регулярность 
множества $\Phi_{\left\{q_{n}\right\}}(\mathfrak{S}(\mathscr{H})) \subseteq \mathscr{K}_{H, 1}$ в случаях А) и Б), а значит, в силу теоремы 2 , каждое входное оптимальное среднее является барицентром по крайней мере одной оптимальной меры.

В случаях А) и Б) состояние $\Omega\left(\Phi_{\left\{q_{n}\right\}}\right)$ является образом барицентра оптимальной меры и, следовательно, лежит в $\Phi_{\left\{q_{n}\right\}}(\mathfrak{S}(\mathscr{H}))$. Для доказательства отсутствия входных оптимальных средних для канала $\Phi_{\left\{q_{n}\right\}}$ в случае В) достаточно показать, что состояние $\Omega\left(\Phi_{\left\{q_{n}\right\}}\right)$ не лежит в $\Phi_{\left\{q_{n}\right\}}(\mathfrak{S}(\mathscr{H}))$.

Поскольку множество $\Phi_{\left\{q_{n}\right\}}(\mathfrak{S}(\mathscr{H}))$ является $\sigma$-выпуклой оболочкой множества $\mathscr{S}_{\left\{q_{n}\right\}}^{1}=\left\{\sigma_{k}\right\}_{k \in \mathbf{Z} \backslash\{0\}}$, предположение $\Omega\left(\Phi_{\left\{q_{n}\right\}}\right) \in \Phi_{\left\{q_{n}\right\}}(\mathfrak{S}(\mathscr{H}))$ означает существование распределения вероятностей $\left\{\pi_{k}\right\}_{k \in \mathbf{Z} \backslash\{0\}}$ такого, что $\Omega\left(\Phi_{\left\{q_{n}\right\}}\right)=\sum_{k \in \mathbf{Z} \backslash\{0\}} \pi_{k} \sigma_{k}$. Сравнивая данное разложение с выражением (10) для состояния $\Omega\left(\Phi_{\left\{q_{n}\right\}}\right)$ в случае $\left.\mathrm{B}\right)$, получаем

$$
q_{k}\left(\pi_{-k}+\pi_{k}\right)=\frac{\exp \left(-\lambda_{\left\{q_{n}\right\}}^{*} / q_{k}\right)}{1+\sum_{j=1}^{+\infty} \exp \left(-\lambda_{\left\{q_{n}\right\}}^{*} / q_{j}\right)} \quad \forall k \in \mathbf{N}
$$

и, следовательно,

$$
\sum_{k=1}^{+\infty} \frac{1}{q_{k}} \exp \left(-\frac{\lambda_{\left\{q_{n}\right\}}^{*}}{q_{k}}\right)=1+\sum_{k=1}^{+\infty} \exp \left(-\frac{\lambda_{\left\{q_{n}\right\}}^{*}}{q_{k}}\right) .
$$

Последнее равенство означает, что в неравенстве (15) в работе [10] для $\mathfrak{H}$-оператора $H$, определенного формулой (6), на самом деле имеет место равенство. В [10] показано, что равенство в неравенстве (15) равносильно неравенству $h_{*}(H) \geqslant h=1$, которое, в свою очередь, равносильно неравенству $F_{\left\{q_{n}\right\}}\left(\lambda_{\left\{q_{n}\right\}}^{*}\right) \geqslant 1$, противоречашему определению случая В).

Если $\lambda_{\left\{q_{n}\right\}}^{*}=\mathrm{g}(H)=0$, то из $(10)$ следует $\mathrm{d}\left(\Omega\left(\Phi_{\left\{q_{n}\right\}}\right)=0\right.$, а значит, в силу предложения $2, \Phi_{\left\{q_{n}\right\}}$ является $\mathrm{CE-каналом.}$

Предположим, что энтропия непрерывна на множестве $\overline{\mathrm{co}}\left(\mathscr{S}_{\left\{q_{n}\right\}}^{1}\right)$. Рассмотрим последовательность состояний

$$
\rho_{n}=\left(1-\sum_{k=1}^{n} q_{k} \pi_{k}\right)|0\rangle\left\langle 0\left|+\sum_{k=1}^{n} q_{k} \pi_{k}\right| k\right\rangle\langle k|=\frac{1}{2} \sum_{k=1}^{n} \pi_{k}\left(\sigma_{k}+\sigma_{-k}\right),
$$

где $\left\{\pi_{k}=q_{k}^{-1}\left(\sum_{k=1}^{n} q_{k}^{-1}\right)^{-1}\right\}_{k=1}^{n}-$ распределение вероятностей. Нетрудно видеть, что эта последовательность лежит в со $\left(\mathscr{S}_{\left\{q_{n}\right\}}^{1}\right)$ и сходится к чистому состоянию $|0\rangle\langle 0|$. В силу предположения о непрерывности энтропии $\lim _{n \rightarrow+\infty} H\left(\rho_{n}\right)=0$, откуда следует

$$
\lim _{n \rightarrow+\infty} n\left(q_{k} \pi_{k}\left(-\log \left(q_{k} \pi_{k}\right)\right)\right)=\lim _{n \rightarrow+\infty} n f\left(\sum_{k=1}^{n} q_{k}^{-1}\right)=0,
$$

где $f(x)=x^{-1} \log x$. Поскольку функция $f(x)$ убывает при больших $x$, из очевидного неравенства $\sum_{k=1}^{n} q_{k}^{-1} \leqslant n q_{n}^{-1}$ и (13) следует $\lim _{n \rightarrow+\infty} \nu_{n}=0$, 
где $\nu_{n}=n f\left(n q_{n}^{-1}\right)=q_{n} \log \left(n q_{n}^{-1}\right)$. Поэтому для произвольного $\lambda>0$ имеем

$$
\left(\frac{q_{n}}{n}\right)^{\lambda / \nu_{n}}=\exp \left(-\frac{\lambda}{q_{n}}\right)
$$

откуда следует $\lambda_{\left\{q_{n}\right\}}^{*}=0$. Предложение 3 доказано.

Наиболее интересный случай в приведенном выше предложении случай В), в котором канал $\Phi_{\left\{q_{n}\right\}}$ проявляет существенно бесконечномерные свойства. Пример последовательности $\left\{q_{n}\right\}$, соответствующей этому случаю, рассмотрен в конце подраздела 3.1 в [11].

Пример 1 можно обобшить, если вместо множества $\mathscr{S}_{\left\{q_{n}\right\}}^{1}$ рассмотреть множество $\mathscr{S}_{\left\{q_{n}\right\}}^{\varepsilon}$ при произвольном $\varepsilon$ из $[0,1]$ (см. [11]).

5. Об одном классе каналов. В этом пункте рассмотрен нетривиальный класс каналов, разрушаюших сцепленность, которые обобщают пример из [2]. Для каналов этого класса $\chi$-пропускная способность и минимальная выходная энтропия определяются явно, а также существует простое необходимое и достаточное условие непрерывности энтропии на замыкании вьходного множества (СЕ-свойства).

Пусть $G$ - компактная группа, $\left\{V_{g}\right\}$ - унитарное представление $G$ в $\mathscr{H}^{\prime}, M(d g)$ - положительная операторнозначная мера на $G$ такая, что множество вероятностных мер $\{\operatorname{Tr} \rho M(\cdot)\}_{\rho \in \mathfrak{S}(\mathscr{H})}$ слабо плотно во множестве всех вероятностных мер на $G$.

Для произвольного состояния $\sigma$ из $\mathfrak{S}\left(\mathscr{H}^{\prime}\right)$ рассмотрим канал

$$
\Phi_{\sigma}(\rho)=\int_{G} V_{g} \sigma V_{g}^{*} \operatorname{Tr} \rho M(d g)
$$

Пусть $\omega\left(G, V_{g}, \sigma\right)=\int_{G} V_{g} \sigma V_{g}^{*} \mu_{H}(d g)$, где $\mu_{H}$ - мера Хаара на $G$. Из предположения слабой плотности множества $\{\operatorname{Tr} \rho M(\cdot)\}_{\rho \in \mathcal{S}(\mathscr{H})}$ во множестве всех вероятностных мер на $G$ следует, что $\overline{\Phi_{\sigma}(\mathfrak{S}(\mathscr{H}))}=$ $\overline{\mathrm{co}}\left\{V_{g} \sigma V_{g}^{*}\right\}_{g \in G}$. Поэтому, используя предложение 10 в [11], нетрудно показать справедливость следующего утверждения.

Предложение 4. $\chi$-пропускная способность канала $\Phi_{\sigma}$ определяется выражением

$$
\bar{C}\left(\Phi_{\sigma}\right)=H\left(\sigma \| \omega\left(G, V_{g}, \sigma\right)\right) \text {. }
$$

$\operatorname{Ecлu} \bar{C}\left(\Phi_{\sigma}\right)<+\infty, \operatorname{mo} \Omega\left(\Phi_{\sigma}\right)=\omega\left(G, V_{g}, \sigma\right) u H_{\min }\left(\Phi_{\sigma}\right)=H(\sigma)$.

Канал $\Phi_{\sigma}$ является СЕ-каналом тогда и только тогда, когда

$$
H\left(\omega\left(G, V_{g}, \sigma\right)\right)<+\infty \text {. }
$$

$B$ этом случае $\bar{C}\left(\Phi_{\sigma}\right)=H\left(\omega\left(G, V_{g}, \sigma\right)\right)-H(\sigma)=H\left(\Omega\left(\Phi_{\sigma}\right)\right)-H_{\min }\left(\Phi_{\sigma}\right)$.

Если канал $\Phi_{\sigma}$ является CE-каналом, то, в силу следствия 2 , достаточным условием существования оптимальной меры является наличие 
по крайней мере одного входного оптимального среднего. Однако именно это условие может быть не выполнено, как показывает следующий пример.

П р и м е р 2. Пусть $G=\mathbf{T}$ - одномерная группа вращений (тор), представленная в виде полуинтервала $[0,2 \pi)$. В этом случае мера Хаара - это нормированная мера Лебега на $[0,2 \pi)$. Пусть $\left\{V_{g}\right\}_{g \in G}-$ группа сдвигов в $\mathscr{H}^{\prime}=\mathscr{L}_{2}(\mathbf{T})$ и $M(d g)$ - спектральная мера оператора умножения на независимую переменную в $\mathscr{H}=\mathscr{L}_{2}(\mathbf{T})$. Нетрудно видеть, что предположение о слабой плотности в этом случае выполнено, а значит, все утверждения предложения 4 имеют место для соответствующего канала $\Phi_{\sigma}$ при произвольном состоянии $\sigma$ из $\mathfrak{S}\left(\mathscr{L}_{2}(\mathbf{T})\right)$. Предположим, что $\sigma=|\varphi\rangle\langle\varphi|$, где $\varphi-$ произвольная функция из $\mathscr{L}_{2}(\mathbf{T})$ с единичной нормой. Тогда $\omega\left(G, V_{g},|\varphi\rangle\langle\varphi|\right)=(2 \pi)^{-1} \int_{0}^{2 \pi}\left|\varphi_{x}\right\rangle\left\langle\varphi_{x}\right| d x$, где $\varphi_{x}(t)=\varphi(t-x)$. Этот канал был первоначально использован в [2] как пример канала, разрушающего сцепленность, который не имеет канонического представления с чисто атомической положительной операторнозначной мерой и, следовательно, не имеет представления Крауса с операторами ранга 1.7) В настоящей статье этот канал рассматривается как пример CE-канала, который демонстрирует свойства, характерные для бесконечномерных каналов.

Используя предложение 4, нетрудно получить (см. также [2]), что

$$
\bar{C}\left(\Phi_{|\varphi\rangle\langle\varphi|}\right)=H\left(\omega\left(\mathbf{T}, V_{g},|\varphi\rangle\langle\varphi|\right)\right)=-\sum_{k=-\infty}^{+\infty}\left|\varphi_{k}\right|^{2} \log \left|\varphi_{k}\right|^{2},
$$

где $\left\{\varphi_{k}=(2 \pi)^{-1} \int_{0}^{2 \pi} e^{-\mathbf{i} x k} \varphi(x) d x\right\}_{k \in \mathbf{Z}}-$ множество коэффициентов Фурье функции $\varphi$, и что из конечности ряда (14) следует, что $\Phi_{|\varphi\rangle\langle\varphi|}$ есть $\mathrm{CE}$-канал и

$$
\Omega\left(\Phi_{|\varphi\rangle\langle\varphi|}\right)=\omega\left(\mathbf{T}, V_{g},|\varphi\rangle\langle\varphi|\right)=\sum_{k=-\infty}^{+\infty}\left|\varphi_{k}\right|^{2}\left|\tau_{k}\right\rangle\left\langle\tau_{k}\right|,
$$

где $\left\{\tau_{k}(x)=\exp (\mathrm{i} k x)\right\}_{k \in \mathbf{Z}}$ - тригонометрический базис в $\mathscr{L}_{2}(\mathbf{T})$. Интересно отметить, что свойства канала $\Phi_{|\varphi\rangle\langle\varphi|}$ определяются скоростью убывания коэффициентов Фурье функции $\varphi$.

Предположим, что для некоторой функции $\varphi$ ряд (14) конечен и, следовательно, $\Phi_{|\varphi\rangle\langle\varphi|}-\mathrm{CE}$-канал. В силу предложения $4 H_{\min }\left(\Phi_{|\varphi\rangle\langle\varphi|}\right)=0$, несмотря на то, что множество $\Phi_{|\varphi\rangle\langle\varphi|}(\mathfrak{S}(\mathscr{H}))$ не содержит чистых состояний. В силу предложения 1 функции $\chi_{\Phi_{|\varphi\rangle\langle\varphi|}}$ и $\widehat{H}_{\Phi_{|\varphi\rangle\langle\varphi|}}$ ограничены и непрерывны на $\mathfrak{S}(\mathscr{H})$. Тем не менее не сушествует ни оптимальной меры, ни входного оптимального среднего состояния для канала $\Phi_{|\varphi\rangle\langle\varphi|}$

7) Любой конечномерный канал, разрушающий сцепленность, имеет представление Крауса с операторами ранга 1 [16]. 
(поскольку в противном случае следствие 2 приводит к существованию оптимальной меры). Непрерывные функции $\chi_{\Phi_{\mid \varphi)\langle\varphi|}}$ и $\widehat{H}_{\Phi_{|\varphi\rangle\langle\varphi|}}$ не достигают соответственно точной верхней грани $\bar{C}\left(\Phi_{|\varphi\rangle\langle\varphi|}\right)<+\infty$ и точной нижней грани $H_{\min }\left(\Phi_{|\varphi\rangle\langle\varphi|}\right)=0$ на некомпактном множестве $\mathfrak{S}(\mathscr{H})$.

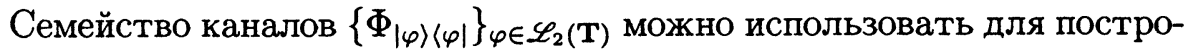
ения еще одного примера, показывающего, что $\chi$-пропускная способность не является непрерывной функцией канала в бесконечномерном случае (см. [17]).

Нетрудно видеть, что для произвольной последовательности $\left\{\left|\varphi_{n}\right\rangle\right\}$ единичных векторов из $\mathscr{L}_{2}(\mathrm{~T})$, сходящейся к вектору $\left|\varphi_{*}\right\rangle$ из $\mathscr{L}_{2}(\mathrm{~T})$, последовательность $\sup _{\rho \in \mathfrak{S}(\mathscr{H})}\left\|\Phi_{\left|\varphi_{n}\right\rangle\left\langle\varphi_{n}\right|}(\rho)-\Phi_{\left|\varphi_{*}\right\rangle\left\langle\varphi_{*}\right|}(\rho)\right\|_{1}$ сходится к нулю. Это значит, что $\varphi \mapsto \Phi_{|\varphi\rangle\langle\varphi|}-$ непрерывное отображение из $\mathscr{L}_{2}(\mathrm{~T})$ во множество всех каналов с топологией равномерной сходимости.

Пусть $\left\{\varphi_{n}\right\}-$ последовательность функций из $\mathscr{L}_{2}(\mathbf{T})$ с коэффициентами Фурье

$$
\left(\varphi_{n}\right)_{k}= \begin{cases}\sqrt{1-q_{n}}, & k=0, \\ \sqrt{q_{n} / n}, & 0<k \leqslant n, \\ 0 & \text { для других } k,\end{cases}
$$

где $\left\{q_{n}\right\}-$ последовательность чисел из $(0,1)$ такая, что

$$
\lim _{n \rightarrow+\infty} q_{n} \log n=C>0 .
$$

Ясно, что последовательность $\left\{\varphi_{n}\right\}$ сходится в $\mathscr{L}_{2}(\mathbf{T})$ к функции $\varphi_{*}(x) \equiv 1$. В силу вышесказанного последовательность каналов $\left\{\Phi_{\left|\varphi_{n}\right\rangle\left\langle\varphi_{n}\right|}\right\}$ равномерно сходится к каналу $\Phi_{\left|\varphi_{*}\right\rangle\left\langle\varphi_{*}\right|}$, У которого $\Phi_{\left|\varphi_{*}\right\rangle\left\langle\varphi_{*}\right|}(\rho)=\left|\varphi_{*}\right\rangle\left\langle\varphi_{*}\right|$ для всех $\rho$ из $\mathfrak{S}(\mathscr{H})$ и, следовательно, $\bar{C}\left(\Phi_{\left|\varphi_{*}\right\rangle\left\langle\varphi_{*}\right|}\right)=0$. В силу (14) имеем

$$
\bar{C}\left(\Phi_{\left|\varphi_{n}\right\rangle\left\langle\varphi_{n}\right|}\right)=h_{2}\left(q_{n}\right)+q_{n} \log n \quad \forall n \in \mathbf{N},
$$

и, следовательно, $\lim _{n \rightarrow+\infty} \bar{C}\left(\Phi_{\left|\varphi_{n}\right\rangle\left\langle\varphi_{n}\right|}\right)=C>0$.

Автор благодарен А.С.Холево за помощь в процессе работы над данной статьей, а также рецензенту, сделавшему ряд полезных замечаний.

\section{СПИСОК ЛИТЕРАТУРЫ}

1. Браттели У., Робинсон Д. Операторные алгебры и квантовая статистическая механика. М: Мир, 1982, $511 \mathrm{c.}$

2. Вернер P. Ф., Холево А.C., Широков $M . E$. О понятии сцепленности в гильбертовых пространствах. - Успехи матем. наук, 2005, т. 60, № 2, с. 153-154.

3. Холево А.C. Введение в квантовую теорию информации. М.: МЦНМО, 2002, $126 \mathrm{c}$. 
4. Холево А.С. Статистическая структура квантовой теории. М.-Ижевск: ИКИ, 2003, $191 \mathrm{c}$

5. Холево А. С. Классическая пропускная способность квантовых каналов с ограничениями. - Теория вероятн. и ее примен., 2003, т. 48, в. 2, с. 359-374.

6. Холево A.C., ШИироков M.E. Непрерывные ансамбли и классическая пропускная способность квантовых каналов бесконечной размерности. - Теория вероятн. и ее примен., 2005 , т. 50 , в. 1 , с. $98-114$.

7. Колмогоров $A . H$., Фомин C.B. Элементы теории функций и функционального анализа. М.: Наука, 1989, 623 с.

8. Иоффе А.Д., Тихомиров В.М. Теория экстремальных задач. М.: Наука, 1974, $479 \mathrm{c}$.

9. Широков M.E. О свойствах квантовых каналов, связанньгх с классической пропускной способностью. - Теория вероятн. и ее примен., 2007, т. 52 , в. 2, с. 301335 .

10. ІШироков M. Е. Энтропийные характеристики подмножеств состояний. I. - Изв. PAH, 2006, т. 70, №6, c. 193-222.

11. ІШироков M. Е. Энтропийные характеристики подмножеств состояний. II. - Изв. PAH, 2006, т. 71, № 1, c. 187-224.

12. Alfsen E.M. Compact Convex Sets and Boundary Integrals. New York-Heidelberg: Springer-Verlag, 1971, $210 \mathrm{p}$.

13. Dell'Antonio G. F. On the limits of sequences of normal states. - Comm. Pure Appl. Math., 1967 , v. 20, p. 413-429.

14. Lindblad G. Expectations and entropy inequalities for finite quantum systems. Comm. Math. Phys., 1974, v. 39, № 2, p. 111-119.

15. Lindblad $G$. Completely positive maps and entropy inequalities. - Comm. Math. Phys., 1975, v. 40, № 2, p. 147-151.

16. Horodecki M., Shor P. W., Ruskai M.B. Entanglement breaking channels. - Rev. Math. Phys., 2003, v. 15, №6, p. 629-641; arxiv: quant-ph/0302031.

17. Shirokov M.E. The Holevo capacity of infinite dimensional channels and the additivity problem. - Comm. Math. Phys., 2006, v. 262, №1, p. 137-159; arxiv: quant$\mathrm{ph} / 0408009$.

18. Schumacher B., Westmoreland M. Optimal signal ensembles. - Phys. Rev. A, 2001, v. 63,022308 ; arxiv: quant-ph/9912122.

19. Parthasarathy K. Probability Measures on Metric Spaces. New York-London: Academic Press, 1967, 276 p.

20. Wehrl A. General properties of entropy. - Rev. Modern Phys., 1978, v. 50, №2, p. 221-260.

Поступила в редакцию

5.X.2006

Исправленный вариант

15.VI.2008 